\title{
Kinetically-Induced Hexagonality in Chemically Grown Silicon Nanowires
}

\author{
Xiaohua Liu and Dunwei Wang $(\bowtie)$ \\ Department of Chemistry, Merkert Chemistry Center, Boston College, 2609 Beacon St., Chestnut Hill, MA 02467, USA \\ Received: 8 April 2009 / Revised: 11 May 2009 / Accepted: 12 May 2009 \\ CTsinghua University Press and Springer-Verlag 2009. This article is published with open access at Springerlink.com
}

\begin{abstract}
Various silicon crystal structures with different atomic arrangements from that of diamond have been observed in chemically synthesized nanowires. The structures are typified by mixed stacking mismatches of closely packed Si dimers. Instead of viewing them as defects, we define the concept of hexagonality and describe these structures as Si polymorphs. The small transverse dimensions of a nanowire make this approach meaningful. Unique among the polymorphs are cubic symmetry diamond and hexagonal symmetry wurtzite structures. Electron diffraction studies conducted with $\mathrm{Au}$ as an internal reference unambiguously confirm the existence of the hexagonal symmetry Si nanowires.

Cohesive energy calculations suggest that the wurtzite polymorph is the least stable and the diamond polymorph is the most stable. Cohesive energies of intermediate polymorphs follow a linear trend with respect to their structural hexagonality. We identify the driving force in the polymorph formations as the growth kinetics. Fast longitudinal elongation during the growth freezes stacking mismatches and thus leads to a variety of Si polymorphs. The results are expected to shed new light on the importance of growth kinetics in nanomaterial syntheses and may open up ways to produce structures that are uncommon in bulk materials.
\end{abstract}

\section{KEYWORDS}

Silicon nanowires, hexagonality, polytypes, cohesive energy, chemical vapor deposition, kinetics

Physical properties of semiconductors are highly sensitive to their crystal structures. Electronic band gaps and phonon frequencies of $\mathrm{SiC}$, for instance, are drastically different for zinc blende ( $Z B$, cubic symmetry) and wurtzite (WZ, hexagonal symmetry) crystals [1]. Similar results have been obtained for other binary semiconductors, primarily III-V and II -VI octet compounds [2]. Theoretical studies predict that hexagonal symmetry Si crystals exhibit reduced electronic band gaps compared with that of the more commonly studied diamond polymorph [3]. In the quest for electronic materials to sustain device scaling, such unique properties may potentially rejuvenate the interest in $\mathrm{Si}$, using its wurtzite polymorph to replace the currently dominant diamond one. It is a particularly appealing prospect given the massive infrastructure for Si-based electronics.

Unlike binary semiconductors, reports of stable

Address correspondence to dunwei.wang@bc.edu 
WZ Si under ambient conditions are scarce [4-6]. Despite the rareness of WZ Si, Sánchez-Dehesa argued that the total energy difference between the two polymorphs (diamond and WZ) of Si is insignificant; instead, the likely reason responsible for the stability of the diamond polymorph is the electrostatic energy [7]. Using the objective molecular dynamics (OMD) approach, Zhang et al. calculated energetic stabilizations of WZ Si nanowires (SiNWs) [8]. Nevertheless, it remains unclear whether the same conclusions apply to SiNWs with diameters $>10$ $\mathrm{nm}$. Recently, Morral et al. reported that WZ SiNWs were obtained using chemical vapor deposition (CVD) synthesis [9-11]. They proposed that the surface energy plays the key role in stressing the Si lattice, leading to hexagonal Si crystals. However, the key experimental evidence in identifying crystal structures-diffraction patterns-is missing in their reports. In this communication, we provide unambiguous structural evidence to show that WZ SiNWs can indeed be obtained using CVD synthesis. By carefully comparing the electron diffraction (ED) patterns of WZ and diamond SiNWs, we highlight the similarities and difference of the two structures. Cohesive energy calculations predict the existence of a variety of Si polymorphs that manifest the transition from diamond to WZ in SiNWs. We provide experimental evidence to support this hypothesis. Our results suggest that the growth kinetics plays a critical role in inducing these unusual structures.

\section{Experimental}

\subsection{SiNWs growth}

SiNWs were synthesized in a homebuilt CVD system with automatic pressure, temperature, and gas flow controls, using protocols that were similar to those in the Refs. [12, 13]. Au nanoparticles (10-100 nm, British Biocell International) served as growth seeds. The reaction was performed at $475{ }^{\circ} \mathrm{C}$ with $60 \mathrm{sccm}$ (standard cubic centimeters per minute) of $\mathrm{SiH}_{4}$ gas flow (10\% in He, Airgas) and $30 \mathrm{sccm}$ of $\mathrm{H}_{2}$ co-flow. To achieve different growth kinetics, the pressure was varied from 5 to 50 Torr [12].

\subsection{Transmission electron microscopy}

As-synthesized SiNWs were studied using a transmission electron microscope (TEM, JOEL JEM 2010F) without post-growth treatments. Electron diffraction patterns (EDPs) were either recorded on Kodak TEM negative films or digitized with a Gatan CCD camera. For camera length calibration purposes, a $6 \mathrm{~nm}$ gold film was coated onto the carbon film TEM grids in an e-beam evaporator (Kurt J. Lesker Company). With the gold film as an internal reference, the EDPs from SiNWs were readily indexed to identify the crystal structures. A throughfocus series of high-resolution electron micrographs (HREM) were recorded with the CCD camera and compared with simulated images using the EMS package [14]. All fast Fourier transform (FFT) and inverse FFT (iFFT) calculations were performed using the DigitalMicrograph ${ }^{\mathrm{TM}}$ (Gatan Inc, Version 3.7.4) software.

\section{Results and discussion}

\subsection{Dependence of cohesive energy on hexagonality}

In crystal chemistry, polytypism refers to varying stacking sequences of close-packed layers [15]. For example, when Si-Si dimers are viewed as one unit, a diamond structure assumes the stacking sequence of $\cdots A B C A B C \cdots$. That is, any given layer is preceded and succeeded by different layers. Any of the $\cdots A B C A B C \cdots$ layers may be defined as $C$, denoting a cubic layer (Fig. 1(a)). The diamond structure may thus be represented


WZ structure is $\cdots A B A B A B \cdots$. The layers preceding and succeeding any given layer are identical. A layer that is adjacent to two identical layers, such as $B$ in $\cdots A B A \cdots$, may be defined as $h$, denoting a hexagonal layer. This leads to WZ being represented as $\cdots h h h h h h \cdots$. Examples of various polytypes and different notations are summarized in Table 1.

We next define hexagonality as the percentage of the $h$ layers in a crystal:

$$
H=\frac{N_{\mathrm{h}}}{N_{\text {total }}}=\frac{N_{\mathrm{h}}}{N_{\mathrm{h}}+N_{\mathrm{c}}}
$$

where $N_{\mathrm{h}}$ refers to the total number of hexagonal 


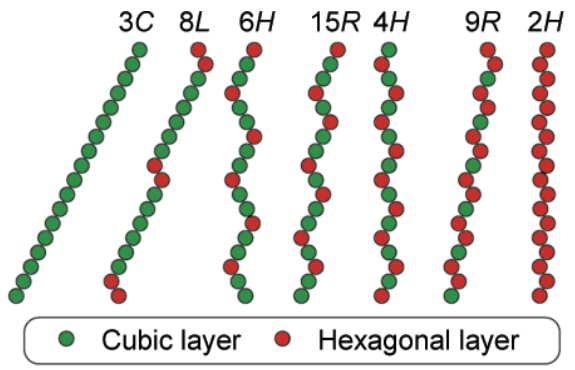

(a)



(b)

Figure 1 Hexagonality and cohesive energies of Si polymorphs. Various Si polymorphs are schematically shown in (a). They are named following the Ramsdell notation. The cohesive energies are plotted in (b) against the hexagonalities of the structure layers and $N_{\mathrm{c}}$ refers to the total number of cubic layers. Under this definition, $H_{\text {wurtzite }}=100 \%$ and $H_{\text {diamond }}=0 \%[1,3]$. The key consequence of this definition is to regard irregular stacking sequences in $\mathrm{Si}$ as new phases rather than defects. Our approach may be justified by the small dimensions across a nanowire $[16,17]$. Similar approaches become impractical when applied to bulk crystals as the number of possible phases will be astronomical. Therefore, it makes more sense to treat the stacking variations as defects in bulk materials. Once defined, cohesive energies of these Si polymorphs can be calculated using an approach recently reported by Glas [18]. This simple approach only looks at the cohesive energy difference as a result of stacking sequence variations. For structures that differ in stacking sequences such as those studied here, this simplification helps highlight the key differences and offers intuitive illustrations of apparently complex crystal structures.

For cohesive energy calculations, we assumed that the distance between adjacent layers is fixed at 0.31 $\mathrm{nm}$ for Si. For any polymorph with arbitrary stacking sequences, its cohesive energy with respect to the ground state may be described as:

$$
\delta E_{\mathrm{p}}=\frac{N_{\mathrm{h}} \delta_{1}-2 n_{\mathrm{c}} \delta_{2}}{N_{\text {total }}}
$$

Here, $\delta_{1}$ refers to the areal energy difference

Table 1 Notations for various Si polymorphs

\begin{tabular}{c|c|c|c|c|c}
\hline Ramsdell notation & Stacking sequence & Jagodzinski notation & Hexagonality $(\%)$ & \multicolumn{2}{|c}{$\delta E_{\mathrm{p}}\left(\mathrm{mJ} / \mathrm{m}^{2}\right)$} \\
\hline $3 C$ & $A B C$ & $(k)_{3}$ & 0 & 0 & 0 \\
\hline $8 H(8 L)[18]$ & $A B A C B A C B$ & $(h h k k k k k k)$ & 25 & $\frac{1}{4} \delta_{1}-\frac{1}{4} \delta_{2}$ & 6.9 \\
\hline $6 H$ & $A B C A C B$ & $(h k k)_{2}$ & 33 & $\frac{1}{3} \delta_{1}-\frac{2}{3} \delta_{2}$ & 8.6 \\
\hline $15 R$ & $A B C A C B C A B A C A B C B$ & $(h k k h k)_{3}$ & 40 & $\frac{2}{5} \delta_{1}-\frac{4}{5} \delta_{2}$ & 10.3 \\
\hline $4 H$ & $A B C B$ & $(h k)_{2}$ & 50 & $\frac{1}{2} \delta_{1}-\delta_{2}$ & 12.8 \\
\hline $9 R$ & $A B A C A C B C B$ & $(h h k)_{3}$ & 67 & $\frac{2}{3} \delta_{1}-\frac{2}{3} \delta_{2}$ & 18.3 \\
\hline $2 H$ & $A B$ & $(h)_{2}$ & 100 & $\delta_{1}$ & 29.3 \\
\hline
\end{tabular}


between the cubic and hexagonal layers and $\delta_{2}$ is the stabilization energy as a result of stacking faults. Adopting $\delta_{1}=29.3 \mathrm{~mJ} / \mathrm{m}^{2}$ and $\delta_{2}=1.8 \mathrm{~mJ} / \mathrm{m}^{2}$, we plotted the cohesive energies of various polymorphs (Fig. 1(b)). As shown, the WZ structure $(2 \mathrm{H})$ is the least stable and the cubic one $(3 C)$ is the most stable stacking. Between these two extremes, the cohesive energy of a variety of polymorphs follows a nearly linear trend with respect to $H$. We next present detailed structural evidence to prove that WZ Si was indeed produced together with the diamond polymorph. This is followed by proof of the hypothesis that intermediate polymorphs should accompany these two polytypes.

\subsection{HRTEM characterization of diamond and wurtzite SiNWs}

The nearly exclusive existence of the Si diamond phase under ambient conditions has been beneficial in the preparation of high quality Si crystals, making today's electronic era possible. The ubiquity has also been enjoyed by researchers in structural characterization. For example, its distinctive features have been conveniently utilized as a standard to calibrate electron microscopes. Prudent approaches should be taken when analyzing nanostructured $\mathrm{Si}$, however, particularly for chemically synthesized samples. In addition to the lattice-fringe-resolved high-resolution (HR) images, the diffraction pattern must be examined with caution using a calibrated camera length, as the difference between various polymorphs can be easily overlooked [4, 5, 9]. The following discussion pertains to SiNWs prepared by the vapor-liquid-solid (VLS) mechenism, evidenced in Figs. 2 (a) and 2 (b). To take Figs. 2 (c) and 2(d): the detailed atomic arrangements (projected structure image along the 3-fold axis, namely 〈111〉 in diamond or $\langle 0001\rangle$ in wurtzite $\mathrm{Si}$,) only differ in the lattice distance, which could be miscalculated without an internal reference. High quality EDPs can help settle potential disputes. As shown in Figs. 2(g) and 2(h), the primary reflections for wurtzite Si fall inside the internal reference diffraction ring of $\mathrm{Au}$ ( $\{111\}$ planes with a spacing of $0.236 \mathrm{~nm}$; for clarity, the diffraction ring is only shown schematically (see Fig. S-1 in the Electronic Supplementary Material
(ESM) for the actual experimental data). By contrast, the diffraction pattern of diamond Si shows primary reflections outside the $\mathrm{Au}$ reference. The weak diffractions inside are forbidden $(1 / 3\{422\})$ but appear as a result of surface terminations, a more significant phenomenon for nanostructures with higher ratios of surface atom (see Fig. S-3 in the ESM) [19, 20]. We emphasize the relative intensity of various reflections.
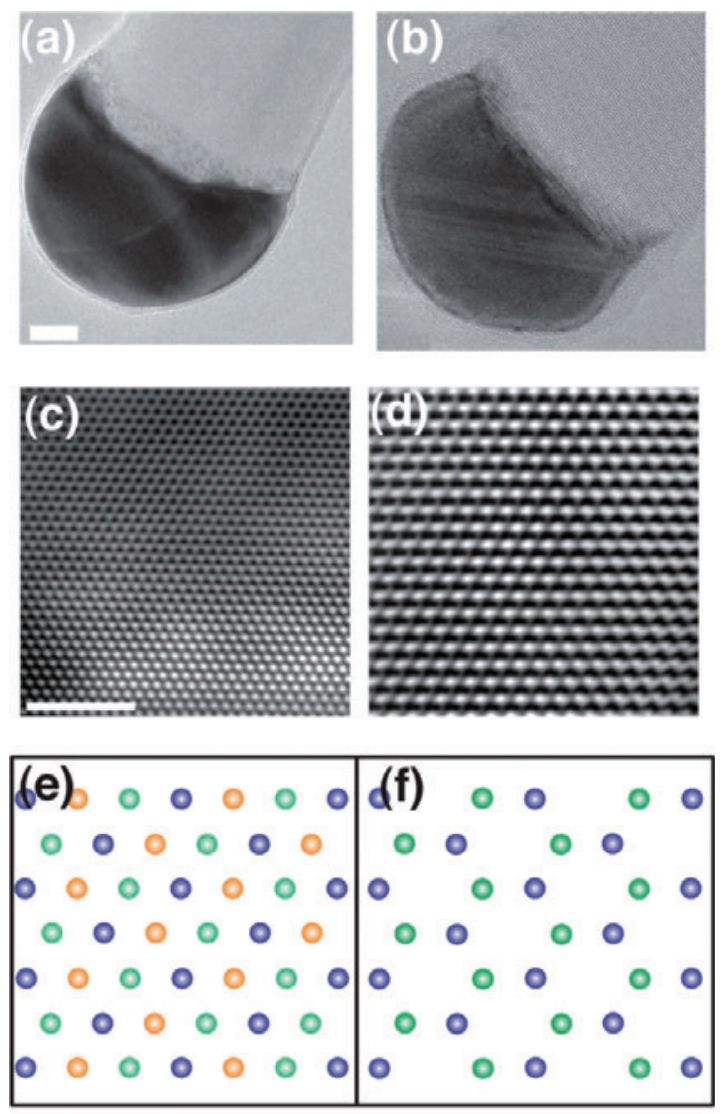

\section{$\circ A \circ B \circ C$}


Figure 2 Structural characterization of diamond (left panels) and wurtzite Si (right panels). TEM micrographs are shown in (a)-(d). The difference is more obvious in HRTEM pictures (c) and (d) under the same magnifications. Scale bars in (a) and (b): $5 \mathrm{~nm}$; in (c) and (d): $2 \mathrm{~nm}$. To illustrate the difference, projected atomic arrangements are shown in (e) and (f). (g) and (h) show their corresponding electron diffraction patterns with the internal reference (Au $\{111\}$ ) schematically highlighted 
The information may be missing or misrepresented in FFT patterns of HR images, an increasingly popular technique adopted by researchers. While convenient in quickly revealing the symmetry of an HR image, the FFT pattern conveys no more information than its parental image, which is sensitive to the imaging conditions of the microscope such as defocus depth and crystal thickness (see Fig. S-2 in the ESM for more discussion).

The difference between the HR images of diamond and WZ Si can be understood by considering the stacking sequence differences. Viewed along the [111] (or [0001] for WZ) zone axis, Figs. 2(e) and 2(f) schematically demonstrate the distinctions in the lattice density. In brief, the large spacing in the $\mathrm{WZ}$ polymorph is due to the lack of $C$ layers in the $\cdots A B A B A B \cdots$ sequence, compared to $\cdots A B C A B C \cdots$ in diamond Si. Although clearly manifested in Figs. 2(c) and 2(d), such difference is ambiguous under different imaging conditions, as shown in the defocus series data (Fig. $\mathrm{S}-2$ in the ESM). A similar defocus series has been demonstrated through simulations in Ref. [9]

\subsection{Polymorphs in SiNWs}

As predicted in Fig. 1, one expects to observe intermediate polymorphs in a system where diamond and WZ ones coexist. Figure 3 shows a few examples of the various polymorphs we observed in this study. The stacking faults are a manifestation of the transition from diamond to $\mathrm{WZ}$, as shown in the cross-section schematics. Similar stacking faults have been reported in binary semiconductor nanostructures [21-23]. Reports for Si are scarce [17, 24]. Worth noting is that a typical growth tends to produce a mixture of various polymorphs (Fig. 4). Although single-phase WZ SiNWs were routinely obtained for a wide range of growth conditions, we failed to observe a condition that produces WZ SiNWs only, i.e., without accompanying polymorphs.

\subsection{Origin of Si polymorphs}

The existence of various Si polymorphs can be accounted for by energetic and/or kinetic factors. Given that the cohesive energy of diamond $\mathrm{Si}$ is the lowest, surface energy must be included in energetic
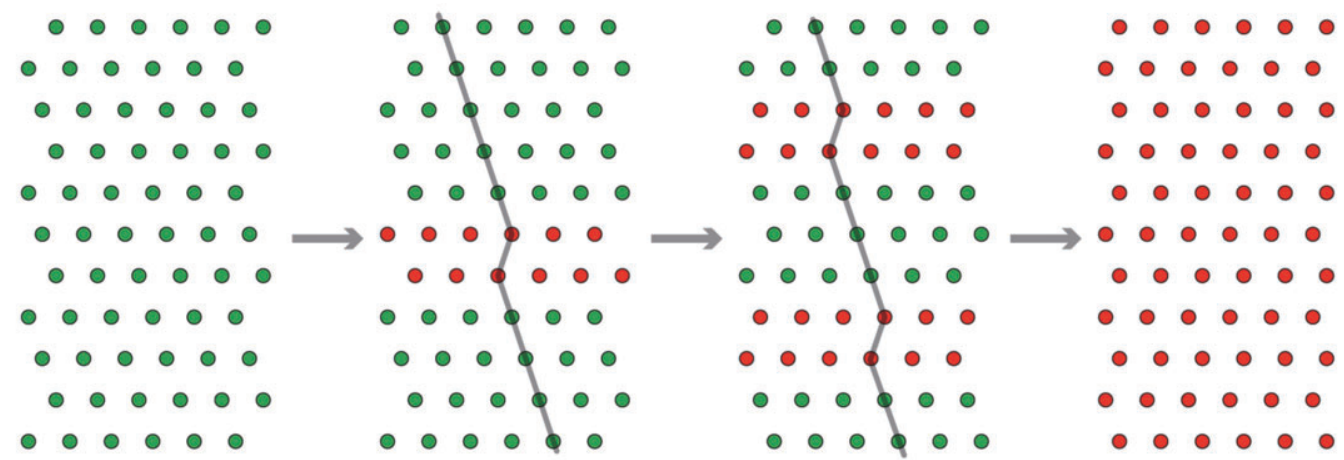

(a)



(b)



(c)

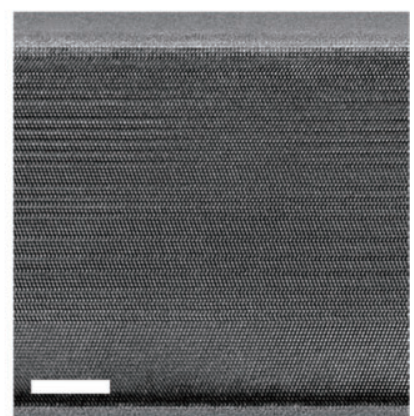

(d)

Figure 3 Microstructures of other polymorph examples. In close-packed Si, a stacking fault appears when a cubic layer is replaced by a hexagonal one, as schematically shown in (a). (b)-(d) show various examples. Scale bars: $5 \mathrm{~nm}$ 
considerations [9]. When the occurrence rate of polymorphs was summarized with respect to the diameter of the NWs, no obvious trend was obtained (Table S-1 in the ESM). This suggests that surface energy is probably not the determining factor. Next, we turned our attention to the growth kinetics. We recently reported that the growth rate of SiNWs is sensitive to the total pressure [12]. Based on this control of growth, we systematically studied the microstructures of SiNWs grown under different rates, from as slow as $\sim 20 \mathrm{~nm} / \mathrm{min}$ to as fast as $\sim 1300$ $\mathrm{nm} / \mathrm{min}$ (Fig. 4). Interestingly, the results showed that fast growth rate favors diversified Si polymorph distributions. We hypothesize the mechanism of $\mathrm{Si}$ polymorph formation as follows.

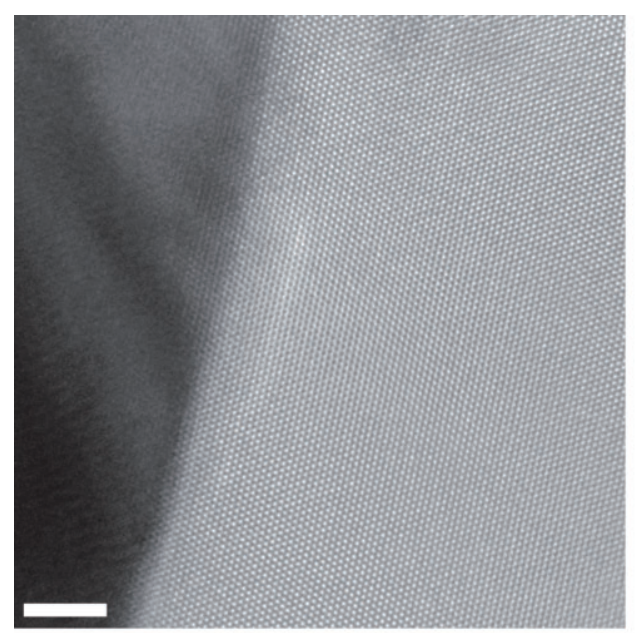

(a)

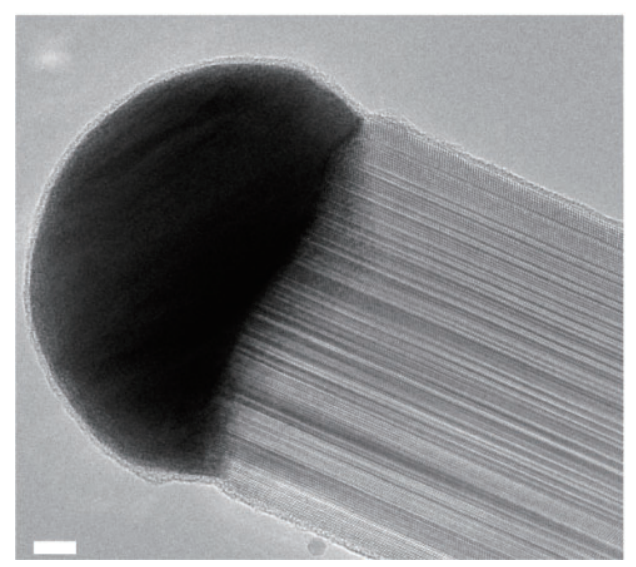

(c)
For a VLS process, the crystal grows as a consequence of solidification from the alloy liquid droplet. Precursor supplies, dissolution in the growth seed and subsequent precipitation result in a delicate steady state. Of these, the precipitation process is of vital importance to the quality of the asgrown crystal. Instabilities within the heterogeneous interface may produce defects such as stacking faults. These faults have a tendency to diffuse transversely to the surface, where they are annihilated, provided that the succeeding $\mathrm{Si}$ atomic additions-hence the longitudinal crystal growth-are slower than that involved in the transverse diffusion. That is, an $\mathrm{NW}$ is expected to grow into a thermodynamically stable phase when the growth kinetics is slow. This is

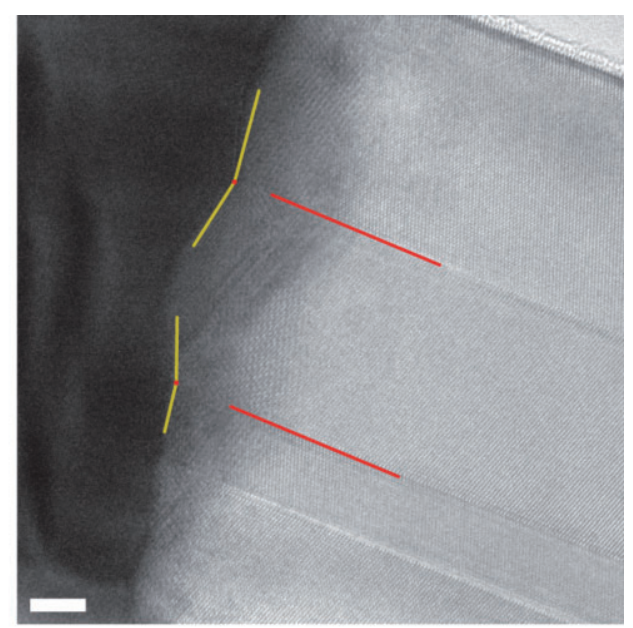

(b)



(d)

Figure 4 The influence of growth kinetics on polymorphs. (a)-(c) HRTEM micrographs of the AuSi heterogeneous interfaces reveal that stacking faults are the result of the interface instabilities, as highlighted by colored lines in (b). Scale bars: $5 \mathrm{~nm}$. The occurrence frequency of Si polymorphs is dependent on the growth kinetics, as shown in (d) 
consistent with our observations that only diamond SiNWs were obtained when the growth rate was sufficiently slow $(20 \mathrm{~nm} / \mathrm{min})$. When the NW growth rate surpasses that of the transverse diffusion of the faults; however, the faults may be trapped. It is thus reasonable to predict that longitudinal stacking faults may form through an NW when the growth kinetics is sufficiently fast.

This indeed has been observed in SiNWs prepared with a fast kinetics (Figs. 3 and 4). For more than 100 SiNWs studied using HRTEM, the faults were exclusively parallel to the longitudinal axis. Furthermore, the mirror planes always coincided with the perturbations of the flat boundary between $\mathrm{Au}$ and $\mathrm{Si}$ when traced to the Au-seed/Si interface (Fig. 4(b), color marks). We note that the polymorphs are remarkably stable once formed. Heating experiments showed that they are preserved after annealing at $900{ }^{\circ} \mathrm{C}$ in $\mathrm{H}_{2}$ (Fig. S-5). Lastly, we note that WZ Si polymorphs appear to be unique in that they appear more often than other polymorphs. More studies are needed to unveil the reason for this.

\section{Conclusions}

By varying the growth kinetics, we obtained various unusual polymorphs of Si. Instead of viewing them as defects, we defined hexagonality as a means to identify them. Among the obtained phases, wurtzite Si can potentially be utilized as a new and attractive candidate for electronic materials with different band structures from that of the diamond polymorph. The results highlight that microstructures of chemically synthesized nanostructures deserve scrutiny as they do not always represent that of their bulk counterparts. Much of the impact of the growth chemistry on the properties of target materials remains to be discovered. The various Si polymorphs obtained in this paper may find a broad range of intriguing applications.

\section{Acknowledgements}

We thank Boston College for financial support. The work was also partially supported by a Department of Defense subcontract from Agiltron. Technical assistance from Y. Lin, Dr. D. Wang, Dr. J. Kong, and Y. -P. Hsieh is gratefully acknowledged.

Electronic Supplementary Material: Supplementary material is available in the online version of this article at http://dx.doi.org/10.1007/s12274-009-9058-z and is accessible free of charge.

\section{References}

[1] Park, C. H.; Cheong, B. H.; Lee, K. H.; Chang, K. J. Structural and electronic properties of cubic, $2 \mathrm{H}, 4 \mathrm{H}$, and 6 H SiC. Phys. Rev. B: Condens. Matter 1994, 49, 44854493.

[2] Yeh, C. -Y.; Lu, Z. W.; Froyen, S.; Zunger, A. Zinc-blendewurtzite polytypism in semiconductors. Phys. Rev. B: Condens. Matter 1992, 46, 10086-10097.

[3] Raffy, C.; Furthmüller, J.; Bechstedt, F. Properties of hexagonal polytypes of group-IV elements from firstprinciples calculations. Phys. Rev. B: Condens. Matter 2002, 66, 075201.

[4] Wentorf, R. H.; Kasper, J. S. Two new forms of silicon. Science 1963, 139, 338-339

[5] Jennings, H. M.; Richman, M. H. A hexagonal (wurtzite) form of silicon. Science 1976, 193, 1242-1243.

[6] Kasper, J. S.; Wentorf, R. H. Hexagonal (wurtzite) silicon. Science 1977, 197, 599.

[7] Sánchez-Dehesa, J.; Vergés, J. A.; Tejedor, C. Diamond structure versus wurtzite structure for silicon. Solid State Commun. 1981, 38, 871-873.

[8] Zhang, D. B.; Hua, M.; Dumitrică, T. Stability of polycrystalline and wurtzite Si nanowires via symmetryadapted tight-binding objective molecular dynamics. J. Chem. Phys. 2008, 128, 084104.

[9] Morral, A. F. I.; Arbiol, J.; Prades, J. D.; Cirera, A.; Morante, J. R. Synthesis of silicon nanowires with wurtzite crystalline structure by using standard chemical vapor deposition. Adv. Mater. 2007, 19, 1347-1351.

[10] Arbiol, J.; Morral, A. F. I.; Estradé, S.; Peiró, F.; Kalache, B.; Cabarrocas, P. R. I.; Morante, J. R. Influence of the (111) twinning on the formation of diamond cubic/diamond hexagonal heterostructures in Cu-catalyzed Si nanowires. J. Appl. Phys. 2008, 104, 064312.

[11] Arbiol, J.; Kalache, B.; Cabarrocas, P. R. I.; Morante, J. R.; Morral, A. F. I. Influence of $\mathrm{Cu}$ as a catalyst on the properties of silicon nanowires synthesized by the vapor 
-solid-solid mechanism. Nanotechnology 2007, 18, 305606.

[12] Zhao, H. Z.; Zhou, S.; Hasanali, Z. H.; Wang, D. W. Influence of pressure on silicon nanowire growth kinetics. J. Phys. Chem. C 2008, 112, 5695-5698.

[13] Cui, Y.; Duan, X. F.; Hu, J. T.; Lieber, C. M. Doping and electrical transport in silicon nanowires. J. Phys. Chem. $B$ 2000, 104, 5213-5216.

[14] Stadelmann, P. A. EMS - A software package for electron diffraction analysis and HREM image simulation in materials science. Ultramicroscopy 1987, 21, 131-145.

[15] Parthé, E. Crystal Chemistry of Tetrahedral Structures; Taylor \& Francis, US, 1964.

[16] Cayron, C.; Den Hertog, M.; Latu-Romain, L.; Mouchet, C.; Secouard, C.; Rouviere, J. -L.; Rouviere, E.; Simonato, J. -P. Odd electron diffraction patterns in silicon nanowires and silicon thin films explained by microtwins and nanotwins. J. Appl. Crystallogr. 2009, 42, 242-252.

[17] Wang, Z. W.; Li, Z. Y. Structures and energetics of indium-catalyzed silicon nanowires. Nano Lett. 2009, 94, 1467-1471.

[18] Glas, F. A simple calculation of energy changes upon stacking fault formation or local crystalline phase transition in semiconductors. J. Appl. Phys. 2008, 104,
093520.

[19] Wei, D. P.; Chen, Q. Metal-catalyzed CVD method to synthesize silicon nanobelts. J. Phys. Chem. C 2008, 112, 15129-15133.

[20] Reyes-Gasga, J.; Gómez-Rodríguez, A.; Gao, X. X.; JoséYacamán, M. On the interpretation of the forbidden spots observed in the electron diffraction patterns of flat Au triangular nanoparticles. Ultramicroscopy 2008, 108, 929-936.

[21] Wang, Z. W.; Daemen, L. L.; Zhao, Y. S.; Zha, C. S.; Downs, R. T.; Wang, X. D.; Wang, Z. L.; Hemley, R. J. Morphology-tuned wurtzite-type ZnS nanobelts. Nat. Mater. 2005, 4, 922-927.

[22] Johansson, J.; Karlsson, L. S.; Dick, K. A.; Bolinsson, J.; Wacaser, B. A.; Deppert, K.; Samuelson, L. Effects of growth conditions on the crystal structure of gold-seeded GaP nanowires. J. Cryst. Growth 2008, 310, 5102-5105.

[23] Hao, Y. F.; Meng, G. W.; Wang, Z. L.; Ye, C. H.; Zhang, L. D. Periodically twinned nanowires and polytypic nanobelts of $\mathrm{ZnS}$ : The role of mass diffusion in vaporliquid-solid growth. Nano Lett. 2006, 6, 1650-1655.

[24] Morales, A. M.; Lieber, C. M. A laser ablation method for the synthesis of crystalline semiconductor nanowires. Science 1998, 279, 208-211. 